\title{
КЛИНИЧЕСКИЙ СЛУЧАЙ ДОФА-ЗАВИСИМОЙ ДИСТОНИИ У МОЛОДОЙ ЖЕНЩИНЫ
}

\author{
Н. А. Белых \\ ${ }^{1}$ Центральная городская клиническая больница № 23, Екатеринбург, Россия \\ 2 Уральский государственный медицинский университет, Екатеринбург, Россия \\ ${ }^{3}$ Уральский федеральный университет имени первого Президента России Б. Н. Ельцина, Екатеринбург, Россия
}

\begin{abstract}
ДОФА-зависимая дистония (ДЗД) - это редкое прогрессирующее генетически гетерогенное заболевание с манисестированием в детском возрасте, в три раза чаще встречающееся у женщин. В статье описан клинический случай синдрома Сегавы у молодой женщины с нижним парапарезом, дистонией стоп (больше справа), нарушением функции ходьбы, поступившей в неотложном порядке в неврологическое отделение с жалобами на выраженное ограничение ходьбы и самообслуживания. В результате дообследования, включающего в себя тест с Леводопой и прямое автоматическое секвенирование по Сенгеру, у пациентки была диагностирована ДЗД. Проведено лечение Леводопой, в ходе которого у пациентки регрессировали клинические симптомы дистонии. В статье представлены особенности течения заболевания и дифференциальной диагностики, а также генетическая детерминированность и тактика лечения.
\end{abstract}

Ключевые слова: ДОФА-зависимая дистония, синдром Сегавы, наследственная дистония

Вклад авторов: Н. А. Белых - сбор материала по заболеванию, дифференциальная диагностика, участие в постановке окончательного диагноза, наблюдение пациента в течение трех лет; М. А. Ахкямова - осмотр пациента, сбор анамнеза заболевания, участие в диагностике, наблюдение пациента в течение трех лет, редактирование рукописи; В. В. Гусев - наблюдение пациента в течение трех лет, назначение диагностических методов исследования, подбор и контроль лечения, предоставление истории болезни пациента для написания статьи, организация этического комитета; О. А. Львова - рекомендации по генетическому исследованию, интерпретация результатов, генетическое консультирование.

Соблюдение этических стандартов: исследование одобрено этическим комитетом Уральского государственного медицинского университета (протокол № 1451/19 от 20 сентября 2019 г.). Получено добровольное информированное согласие на участие пациента в научном исследовании.

$\bowtie$ Для корреспонденции: Никита Алексеевич Белых

ул. Репина, 3, г. Екатеринбург, 620014; nikitabelikh@gmail.com

Статья получена: 14.05.2020 Статья принята к печати: 28.05.2020 Опубликована онлайн: 04.06.2020

DOI: $10.24075 /$ vrgmu.2020.031

\section{A CASE REPORT OF DOPA-RESPONSIVE DYSTONIA IN A YOUNG WOMAN}

Belykh $\mathrm{NA}^{2} \bowtie$, Akhkyamova $\mathrm{MA}^{2}$, Gusev $\mathrm{W}^{1,2,3}$, Lvova OA $\mathrm{A}^{2,3}$

${ }^{1}$ Central Clinical Hospital № 23, Yekaterinburg, Russia

${ }^{2}$ Ural State Medical University, Yekaterinburg, Russia

${ }^{3}$ Yeltsin Ural Federal University, Yekaterinburg, Russia

Dopa-responsive dystonia (DRD) is a rare progressive genetically heterogenous disorder with pediatric onset. DRD is 3 times as prevalent in women than in men. This article reports a clinical case of DRD in a young female presenting with paraparesis, foot dystonia (more pronounced in the right foot) and pronounced walking impairment, who was admitted for emergency treatment to a Neurology Unit. Based on the additional tests, which included a levodopa trial and Sanger sequencing, the patient was diagnosed with DRD. Levodopa caused a considerable improvement of the symptoms. The article describes the clinical features of the disease, talks about its differential diagnosis, genetic predisposition and treatment strategy.

Keywords: DOPA-responsive dystonia, Segawa syndrome, hereditary dystonia

Author contribution: Belykh NA analyzed the literature, made the differential diagnosis, participated in establishing the definitive diagnoses followed the patient up for 3 years. Akhkyamova MA examined the patient, collected her medical history, participated in establishing the diagnosis, followed the patient up for 3 years, wrote the manuscript. Gusev W followed the patient up; ordered diagnostic tests, prescribed treatment and monitored its course; provided the patient's medical history; helped with the application to the Ethics Committee. Lvova OA advised the patient on the genetic test and interpreted its results.

Compliance with ethical standards: the study was approved by the Ethics Committee of Ural State Medical University (Protocol № 1451/19 dated September 20, 2019). The patient gave informed consent to participate in the study.

$\triangle$ Correspondence should be addressed: Nikita A. Belykh Repina, 3, Yekaterinburg, 620014; nikitabelikh@gmail.com

Received: 14.05.2020 Accepted: 28.05.2020 Published online: 04.06.2020

DOI: 10.24075/brsmu.2020.031

ДОФА-зависимая дистония (ДЗД) - одна из форм торзионной дистонии, которую впервые описал японский невролог Сегава в 1971 г. как наследственную прогрессирующую дистонию с выраженными дневными колебаниями сиптоматики [1].

В большинстве случаев дистония начинается с одной ноги в первые 9-10 лет жизни и распространяется на все четыре конечности к концу второго десятилетия [2]. Чаще всего первым симптомом ДОФА-зависимой дистонии является нарушение походки, нередко расцениваемое врачами как детский церебральный паралич [3]. На ранних стадиях симптомы заболевания отсутствуют в утренние часы, но характерны вечерние обострения, которые больше зависят от количества часов бодрствования, чем от физической активности. По мере прогрессирования дистонии симптомы возникают как в утренние, так и в вечерние часы [4].

Различают два типа заболевания: классическую постуральную дистонию (нарушение тонуса мышц во время поддержания позы) и фазическую дистонию (сочетание дистонии при движениях и постуральной дистонии). Описаны также отдельные клинические случаи изолированного тремора рук, постоянной десормации стоп по типу косолапости и дистоний в отдельных мышцах [5]. 
Основным диагностическим тестом при подозрении на ДЗД является назначение препарата Леводопы, после которого происходит видимое улучшение состояния пациента.

Рекомендуемая начальная доза Леводопы составляет 1 мг/кг/день. Ее следует постепенно увеличивать до полного улучшения или до появления побочных эффектов. У большинства пациентов улучшение состояния наступает при дозе 4-5 мг/кг/день. Леводопу следует давать в течение трех месяцев, прежде чем считать испытание неудачным [6].

Для того чтобы отличить ДЗД от других состояний, при которых применение Леводопы оказывает положительный эффект, проводят измерение концентрации птеринов в цереброспинальной жидкости (ЦСЖ). При положительном тесте с Леводопой и повышении концентрации птеринов в ЦСЖ высока вероятность наличия ДЗД [7]. Диагноз подтверждают с помощью молекулярно-генетического тестирования на наличие мутаций в гене GCH1 [8].

\section{Описание клинического случая}

Пациентка с ДЗД проходила обследование и лечение в неврологическом отделении Центральной городской клинической больницы № 23 г. Екатеринбурга с 2015 по 2017 г. Диагноз поставлен по выявленному варианту нуклеотидной последовательности c.248g>a (p.Gly83Asp) в гетерозиготном состоянии методом ПЦР.

Женщина 1982 г. рождения. Из анамнеза: первые симптомы заметили родственники в возрасте 12 лет. «Слабость» в ногах, нарушение походки, усталость мышц ног, нарастающая при ходьбе, «проворачивание правой коленки" и судорожное «подгибание» пальцев стоп при ходьбе, эмоциональное напряжение. Со слов пациентки, иногда возникала слабость в кистях. Ребенка стали регулярно наблюдать и лечить у невролога, Предположительные диагнозы: болезнь Штрюмпеля, парапарез, параплегия. Назначенное лечение (Баклофен, Нейромультивит, Сирдалуд, парафин, массаж, ЛФК) не оказывало эффекта, состояние ухудшалось. На фоне медикаментозной терапии пациентка стала отмечать выраженную слабость, «потеряла контроль над ногами», самостоятельно отменила Баклосен и Сирдалуд, перестала посещать уроки физической культуры, так как не могла выполнять нормативы (особенно любые прыжки от пола).

Анамнез жизни: росла и развилась по возрасту, семья полная. В 3 года перенесла ветряную оспу. Образование

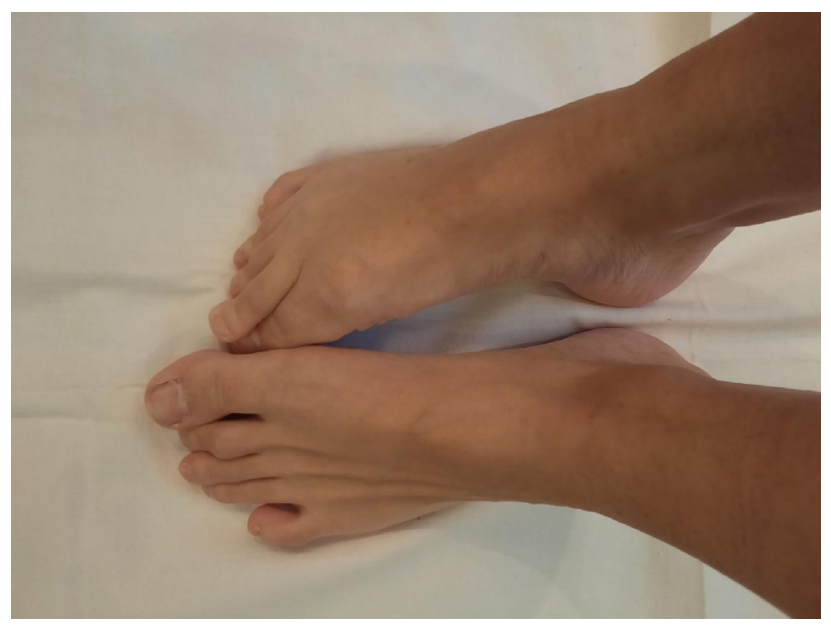

Рис. 1. Дистония правой стопы на фоне отсутствия лечения препаратами Леводопы высшее, экономист, в настоящее время не работает. Наследственность не отягощена, вредных привычек нет. В 2010 г. пациентке проведено родоразрешение путем кесарева сечения. Эпидемиологический и аллергический анамнезы без особенностей. С 1995 г. ребенок-инвалид по ДЗД. С 2000 г. инвалид 2-й группы.

В 2015 г. получила направление на ботулинотерапию. Инъекции ботокса не получала по неизвестным причинам. Произошло нарастание симптоматики - появилось выраженное ограничение ходьбы, самообслуживания; в связи с этим госпитализирована в неотложном порядке в неврологический стационар для дальнейшего обследования.

Данные объективного обследования при поступлении: состояние средней тяжести, кожа бледная, сухая. Пульс ритмичный, 72 уд./мин, АД 130/75, ЧД 16 в минуту. Неврологическое обследование: скандированная речь, сознание ясное, ориентирована правильно. Черепные нервы - без особенностей. Снижение мышечной силы в нижних конечностях до 3,5 баллов дистально, повышение мышечного тонуса по экстрапирамидному тонусу справа (рис. 1), в нижних конечностях - горметония. Реслексы верхних конечностей равные, умеренные, нижних конечностей снижены. Патологических рефлексов нет. Координаторные пробы выполняет неуверенно с двух сторон, в позе Ромберга неустойчива. Менингеальных симптомов нет. Ограничены движения во всех отделах позвоночника. На основании жалоб, анамнеза и клинической картины выставлен предварительный диагноз: энцефалополирадикулонейропатия с нижним парапарезом, дистонией стоп (больше справа), нарушением функции ходьбы. Лабораторные исследования: общий анализ крови, общий анализ мочи, биохимический анализ крови - в пределах нормы. Электрокардиограмма: синусовый ритм 73 уд./мин. Электронейромиография нижних и верхних конечностей: легкая радикулопатия L4L5, S1, C7-C8, Th1. Умеренная аксональная нейропатия локтевых нервов. Легкая аксональная нейропатия правого большеберцового и малоберцовых нервов. Магнитнорезонансная томография головного мозга: кортикальная атрофия 1-й степени. Данных, подтверждающих очаговую и диффузную патологию, не получено (рис. 2). Магнитнорезонансная томография шейного отдела позвоночника от 2007 г.: остеохондроз С3-С6 1-й стадии, мелкий дискоостеофитический комплекс С5-С6. Очаговая патология в спинном мозге не выявлена (рис. 3).

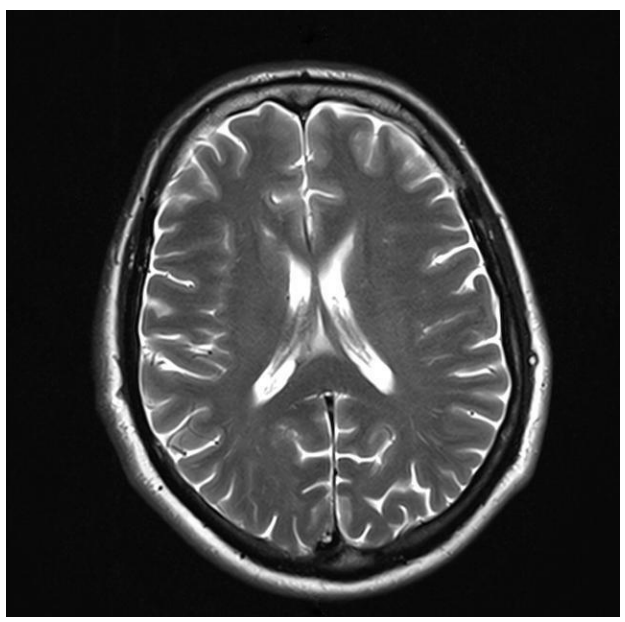

Рис. 2. МРТ головного мозга: кортикальная атрофия 1-й степени, очаговая и диффузная патологии не выявлены 
ДНК-анализ, поиск мутаций в гене GCHI при исследовании от 13 марта 2017 г.: проведено исследование кодирующей последовательности и прилежащих интронных областей гена GCHI, ответственного за торсионную дистонию (DYT5), методом прямого автоматического секвенирования по Сенгеру. Выявлен вариант нуклеотидной последовательности c.248g>a (p.Gly83Asp) в гетерозиготном состоянии. Диагноз «Торсионная дистония» молекулярно-генетическими методами подтвержден.

По совокупности данных, полученных в динамике, диагноз пересмотрен. Окончательный диагноз: G24.8 ДОФА-зависимая дистония с преимущественным поражением обеих стоп, нарушением функции ходьбы. Полирадикулонейропатия на фоне остеохондроза шейного и пояснично-крестцового отделов позвоночника, болевой синдром, моторные нарушения.

Пациентке даны рекомендации: наблюдение невролога по месту жительства, ЛФК ежедневно, дофаминомиметик, вазодилататор, гепатопротектор, поливитамины, ингибитор холинэстеразы. С момента установления окончательного диагноза пациентка постоянно принимала дофаминомиметик до наступления беременности. С момента наступления беременности дозировку снизила в два раза от начальной, отмечала прежнюю эффективность.

\section{Обсуждение клинического случая}

Больные с ДЗД могут иметь четыре формы мутаций гена [9], отвечающего за синтез фермента гуанозинтрифосфатциклогидролазы । (GCH1). Этот фермент участвует в синтезе тетрагидробиоптерина (BН4) кофактора тирозингидроксилазы (ТГ), который превращает L-тирозин в L-ДОФA. B результате снижается содержание дофамина в полосатом теле. У пациентов с аутосомнодоминантным типом наследования патологический ген находится на хромосоме 14 (14qll-q24.3), его продуктом является белок $\mathrm{GCH}$. При аутосомно-рецессивном типе наследования патологический ген расположен на хромосоме 11р15.5 гена ТГ [10].

Распространенность нейрометаболического заболевания составляет 0,5-1 случай на миллион [11], данные могут быть заниженными из-за слабых симптомов у некоторых пациентов. ДЗД в возрасте 4-8 лет сочетается с признаками паркинсонизма и мышечной дистонии, приводящими к изменению походки. Повышение мышечного тонуса в ногах, признаки поражения пирамидных трактов позволяют заподозрить детский церебральный паралич или наследственную спастическую параплегию, что служит причиной ошибок в диагностике [12]. Со временем дистония нижних конечностей переходит в генерализованную дистонию. Характерны суточные колебания выраженности двигательных расстройств с уменьшением после сна и усилением к вечеру. Оценка терапевтического эффекта от приема Леводопы является методом диагностики ДЗД, когда дистония не связана с гипоксической ишемической энцефалопатией [13].
В данном случае у пациентки отмечались снижение мышечной силы в нижних конечностях до 3,5 баллов дистально, повышение мышечного тонуса по экстрапирамидному тонусу справа, расстройство походки с дистоническими движениями нижних конечностей, снижение рефлексов нижних конечностей и неустойчивость в позе Ромберга.

С учетом клинических результатов и улучшения состояния после назначения комбинации Леводопа / Карбидопа был поставлен диагноз ДЗД. Пациентка не имеет клинических симптомов дистонии на сегодняшний день.

\section{ВЫВОДЫ}

ДОФА-зависимая дистония - редкое генетическое заболевание с манифестацией клинических проявлений в детском возрасте. Постановка окончательного диагноза занимает продолжительное время в связи с трудностью диагностики заболевания. Дети с начавшейся дистонией, нарушениями походки зачастую не обращают на себя пристального внимания родителей. Правильно собранный анамнез и целенаправленное неврологическое обследование в поисках суточных изменений симптомов и ответ на терапию являются факторами к постановке верного диагноза. Результаты приведенного выше клинического случая позволят специалистам чаще обращать внимание на данную патологию при обследовании пациента.

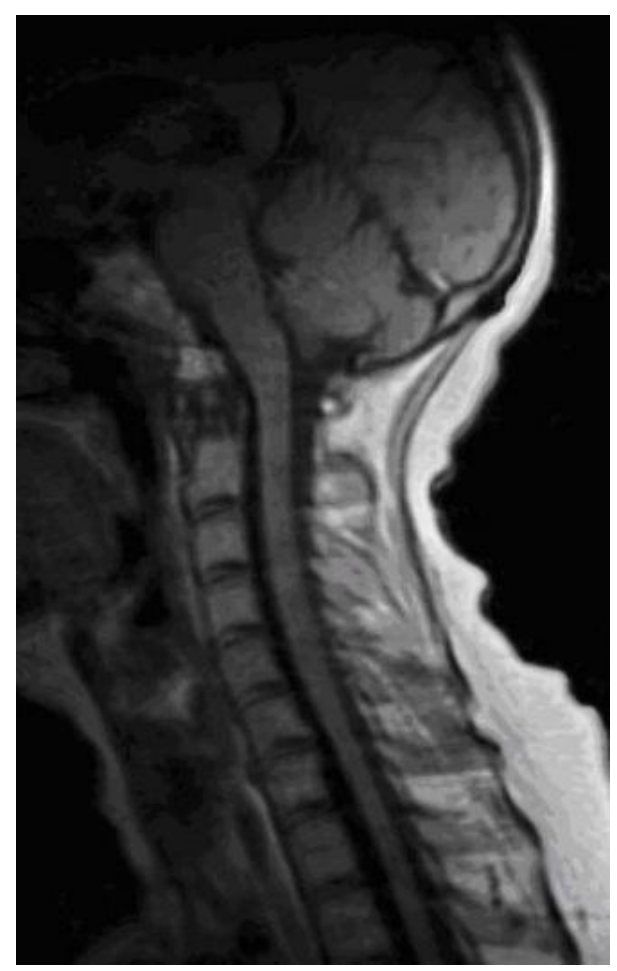

Рис. 3. МРТ шейного отдела позвоночника: остеохондроз С3-С6 1-й стадии, мелкий дискоостеофитический комплекс C5-C6. Очаговая патология в спинном мозге не выявлена 


\section{Литература}

1. Segawa M, Ohmi K, Itoh S, Aoyama M, Hayakawa H. Childhood basal ganglia disease with remarkable response to L-Dopa, hereditary basal ganglia disease with marked diurnal fluctuation. Shinryo. 1971; 24: 667-72.

2. Roubertie A, Mariani LL, Fernandez-Alvarez E, Doummar D, Roze E. Treatment for dystonia in childhood. Eur J Neurol. 2012; 19 (10): 1292-9. DOI: 10.1111/j.1468-1331.2011.03649.x.

3. Lin J, Lumsden DE, Gimeno $\mathrm{H}$, et al. The impact and prognosis for dystonia in childhood including dystonic cerebral palsy: a clinical and demographic tertiary cohort study. J Neurol Neurosurg Psychiat. 2014; 85: 1239-44.

4. Gouider-Khouja N, Kraoua I, Benrhouma H, Fraj N, Rouissi A. Movement disorders in neuro-metabolic diseases. Eur J Paediatr Neurol. 2010; 14 (4): 304-7. DOI: 10.1016/j.ejpn.2009.11.005.

5. Wassenberg $\mathrm{T}$, Schouten Ml, Helmich RC, Willemsen MAAP, Kamsteeg EJ, van de Warrenburg BPC. Autosomal dominant $\mathrm{GCH} 1$ mutations causing spastic paraplegia at disease onset [published online ahead of print, 2020 Apr 1]. Parkinsonism Relat Disord. 2020; 74: 12-15. DOI: 10.1016/j.parkreldis.2020.03.019.

6. Wijemanne S, Jankovic J. Dopa-responsive dystonia - clinical and genetic heterogeneity. Nat Rev Neurol. 2015 Jul; 11 (7): 41424. DOI: $10.1038 /$ nrneurol.2015.86.

7. Nygaard TG, Marsden CD, Duvoisin RC. Dopa-responsive

\section{References}

1. Segawa M, Ohmi K, Itoh S, Aoyama M, Hayakawa H. Childhood basal ganglia disease with remarkable response to L-Dopa, hereditary basal ganglia disease with marked diurnal fluctuation. Shinryo. 1971; 24: 667-72.

2. Roubertie A, Mariani LL, Fernandez-Alvarez E, Doummar D, Roze E. Treatment for dystonia in childhood. Eur J Neurol. 2012; 19 (10): 1292-9. DOI: 10.1111/j.1468-1331.2011.03649.x.

3. Lin J, Lumsden DE, Gimeno H, et al. The impact and prognosis for dystonia in childhood including dystonic cerebral palsy: a clinical and demographic tertiary cohort study. J Neurol Neurosurg Psychiat. 2014; 85: 1239-44.

4. Gouider-Khouja N, Kraoua I, Benrhouma H, Fraj N, Rouissi A. Movement disorders in neuro-metabolic diseases. Eur J Paediatr Neurol. 2010; 14 (4): 304-7. DOI: 10.1016/j.ejpn.2009.11.005.

5. Wassenberg T, Schouten Ml, Helmich RC, Willemsen MAAP, Kamsteeg EJ, van de Warrenburg BPC. Autosomal dominant $\mathrm{GCH} 1$ mutations causing spastic paraplegia at disease onset [published online ahead of print, 2020 Apr 1]. Parkinsonism Relat Disord. 2020; 74: 12-15. DOI: 10.1016/j.parkreldis.2020.03.019.

6. Wijemanne S, Jankovic J. Dopa-responsive dystonia - clinical and genetic heterogeneity. Nat Rev Neurol. 2015 Jul; 11 (7): 41424. DOI: $10.1038 /$ nrneurol.2015.86.

7. Nygaard TG, Marsden CD, Duvoisin RC. Dopa-responsive dystonia. Advances in Neurology. 1988; 50: 377-84

8. Van Hove JL, Steyaert J, Matthiis G, Legius E, Theys P, Wevers R, et al. Expanded motor and psychiatric phenotype in autosomal dominant Segawa syndrome due to GTP cyclohydrolase deficiency. J Neurol Neurosurg Psychiat. 2006; 77: 18-23. Available from: https://DOI.org/10.1136/jnnp.2004.051664.

9. Camargo CHF, Camargos ST, Cardoso FEC, Teive HAG. The genetics of the dystonias - a review based on the new classification of the dystonias. Arquivos de neuropsiquiatria. 2015; 73 (4): 350-8.

10. Lohmann $\mathrm{K}$, Klein $\mathrm{C}$. Update on the genetics of dystonia. Current neurology and neuroscience reports. 2017; 17 (3): 26.

11. Zirn B, Steinberger D, Troidl C, Brockmann K, von der Hagen M, Feiner $\mathrm{C}$, et al. Frequency of $\mathrm{GCH} 1$ deletions in Dopa-responsive dystonia. J Neurol Neurosurg Psychiat. 2008; 79: 183-6. Available from: https://DOI.org/10.1136/jnnp.2007.128413.

12. Lee W-W, Jeon BS. Clinical spectrum of dopa-responsive dystonia and related disorders. Curr Neurol Neurosci Rep. 2014; 14 (7): 461. Available from: https://DOl.org/10.1007/s11910-014-0461-9.

13. Van Egmond ME, Kuiper A, Eggink H, Sinke RJ, Brouwer OF, VerschuurenBemelmans CC, et al. Dystonia in children and adolescents: a systematic review and a new diagnostic algorithm. J Neurol Neurosurg Psychiat. 2015; 86 (7): 774-81. Available from: https://DOl.org/10.1136/jnnp-2014-309106.

dystonia. Advances in Neurology. 1988; 50: 377-84.

8. Van Hove JL, Steyaert J, Matthijs G, Legius E, Theys P, Wevers R, et al. Expanded motor and psychiatric phenotype in autosomal dominant Segawa syndrome due to GTP cyclohydrolase deficiency. J Neurol Neurosurg Psychiat. 2006; 77: 18-23. Available from: https://DOI.org/10.1136/jnnp.2004.051664.

9. Camargo CHF, Camargos ST, Cardoso FEC, Teive HAG. The genetics of the dystonias - a review based on the new classification of the dystonias. Arquivos de neuropsiquiatria. 2015; 73 (4): 350-8.

10. Lohmann K, Klein C. Update on the genetics of dystonia. Current neurology and neuroscience reports. 2017; 17 (3): 26.

11. Zirn B, Steinberger D, Troidl C, Brockmann K, von der Hagen M, Feiner $\mathrm{C}$, et al. Frequency of $\mathrm{GCH} 1$ deletions in Dopa-responsive dystonia. J Neurol Neurosurg Psychiat. 2008; 79: 183-6. Available from: https://DOI.org/10.1136/jnnp.2007.128413.

12. Lee W-W, Jeon BS. Clinical spectrum of dopa-responsive dystonia and related disorders. Curr Neurol Neurosci Rep. 2014; 14 (7): 461. Available from: https://DOI.org/10.1007/s11910-014-0461-9

13. Van Egmond ME, Kuiper A, Eggink H, Sinke RJ, Brouwer OF, VerschuurenBemelmans CC, et al. Dystonia in children and adolescents: a systematic review and a new diagnostic algorithm. J Neurol Neurosurg Psychiat. 2015; 86 (7): 774-81. Available from: https://DOl.org/10.1136/jnnp-2014-309106. 